Mediterránea Ser. Biol. (1987), n. 9. Pág. 41-56

\title{
ACTIVIDAD DEL LAGARTO VERDINEGRO (LACERTA SCHREIBERI) (SAURIA: LACERTIDAE)
}

\author{
por \\ Alfredo Salvador
}

\section{RESUMEN}

En este trabajo se estudia la actividad de Lacerta schreiberi en dos parcelas de Camposagrado (León). En ambas poblaciones se realizaron censos horarios de abril a septiembre. Para calcular el porcentaje de población activa en cada censo, se estimó simultáneamente y por separado para machos, hembras, subadultos y jóvenes el tamño de población en cada parcela.

Los machos están más activos en primavera mientras que en verano apenas se ven. Las hembras presentan menor actividad que los machos y apenas se registran en ellas diferencias estacionales. Los subadultos inician tardíamente su actividad anual aunque están muy activos en todos los meses. En jóvenes se registra escasa actividad.

Los distintos patrones de actividad encontrados en machos, hembras, subadultos y jóvenes se relacionan con los distintos requerimientos de cada uno de ellos.

\section{SUMMARY}

Patterns of activity were studied in two populations of Lacerta schreiberi at Camposagrado (León) from april to september 1984. Population estimates were used to compute the proportion of males, females, subadults and youngs observed during censuses. Males were more active during spring (reproductive season) than in summer. Female activity did not vary between seasons. High activity levels were observed in subadults and activity was very low in youngs during all months.

PALABRAS CLAVE: Actividad, variación intraespecífica, Lacerta schreiberi.

\section{INTRODUCCIÓN}

En general se suelen estimar los patrones de actividad de reptiles contando el número de individuos observados sobre un transecto predeterminado.

(1) Museo Nacional de Ciencias Naturales. J. G. Abascal, 2. 28006 Madrid. 
Los porcentajes observados a distintas horas y en diversas épocas del año se han relacionado con las temperaturas ambientales. Hasta ahora en la península Ibérica solamente se han realizado los tabajos de BUSACK (1976) y SEVA (1982) sobre Acanthodactylus erythrurus y el de PEREZ MELLADO (1983) sobre Podarcis hispanica y Podarcis bocagei.

Recientemente se ha comenzado a estudiar los patrones de actividad intrapoblacionales de varias especies de saurios (SIMON y MIDDENDORF, 1976) (ROSE, 1981) o incluso de comunidades de saurios (CREUSERE y WHITFORD, 1982) encontrándose patrones de actividad muy complejos.

En este trabajo nos proponemos estudiar los patrones de actividad de $\mathrm{La}$ certa schreiberi y examinar si hay variación entre edades y sexos. También queremos conocer si los patrones de actividad varían entre poblaciones. $\mathrm{N}$ os ha parecido interesante conocer los porcentajes de población activa y para ello hemos utilizado estimaciones de población.

\section{AREA DE ESTUDIO}

Para la realización de este estudio se ha elegido una zona situada en los alrededores de Camposagrado (León), a una altitud de $1.200 \mathrm{~m}$. En el mes de marzo de 1984 se establecieron dos parcelas de 0,5 ha. cada una. Ambas parcelas fueron cuadriculadas y cartografiadas mediante una red de estacas dispuestas cada $5 \mathrm{~m}$. entre sí. Por el centro de cada parcela discurre un pequeño arroyo que encharca pequeños prados de Molinia caerulea y Juncus effusus. Hacia afuera se disponen matorrales de Erica arborea, Erica australis y Halimium alyssoides.

\section{MATERIAL Y METODOS}

Para el estudio de la actividad se realizaron en 1984 censos horarios en todos los meses, con un esfuezo similar en ambas parcelas. La parcela AR fue censada los días 10,18 y 25 de abril, 7 y 10 de mayo, 11,15 y 28 de junio, 4 y 12 de julio, 17 y 28 de agosto y 9 de septiembre. La parcela AB se censó los días 13,26 y 30 de junio, 8 y 17 de julio, 25 y 31 de agosto y 11 de septiembre.

Los censos se realizaron durante las horas de sol $(9$ a 19 h.) y cada hora se recorría la parcela por completo anotando los individuos observados, distinguiendo machos, hembras, subadultos y jóvenes. Se ha anotado cada hora la temperatura ambiental, tomándola a $10 \mathrm{cms}$. del suelo a la sombra. La amplitud de la banda de censo fue de $5 \mathrm{~m}$. (separación entre estacas) excepto en las zonas de hierba y junco en que se estrechó a solamente un metro con objeto de detectar mejor a los jóvenes.

Hemos usado nuestras estimaciones de población en ambas parcelas mediante el método de Schnabel de múltiples marcajes y recapturas con el objeto de averiguar el porcentaje de población activa de cada hora distinguiendo machos, hembras, subadultos y jóvenes. En otro trabajo se presentarán los resultados sobre estructura y densidad de población.

Para averiguar si difiere la actividad en ambas parcelas hemos calculado los porcentajes medios por hora en cada mes y en cada parcela, distinguiendo también machos, hembras, subadultos y jóvenes. La comparación se ha reali- 
zado basándonos en la mayor actividad registrada en cada mes y por medio de test de chi-cuadrado.

\section{RESULTADOS}

\section{FENOLOGÍA}

En visitas realizadas a las parcelas en días previos a los primeros censos se comprobó que los primeros individuos observados eran machos ( 2 de abril). Tres días después comienzan a verse los jóvenes. Los censos del día 10 de abril revelan la presencia de hembras y subadultos. Durante la primavera la actividad de los lagartos comienza a partir de las $10 \mathrm{~h}$. con temperaturas de $10^{\circ} \mathrm{C}$. o superiores. A partir del 15 de junio la actividad comienza de las $9 \mathrm{~h}$. y con temperaturas que oscilan entre $12^{\circ}$ y $19^{\circ} \mathrm{C}$.

$\mathrm{La}$ actividad es continua hasta finales de agosto en que dejan de verse adultos y a primeros de septiembre ya sólo se ven algunos subadultos. Cuando comienzan a nacer las crías, a partir del 9 de septiembre, ya no hay adultos ni subadultos.

\section{ACTIVIDAD DE MACHOS}

Las figuras 1 y 2 muestran los resultados referentes a machos. Los censos de abril y mayo muestran que la actividad de los machos es significativamente mayor que en ninguna otra época (cuadro 1). En estos meses se llega a observar a las horas de mayor actividad del 18 al $72 \%$ del total de machos en la parcela AB y del 11 al $35 \%$ en la parcela AR.

\section{CUADRO 1}

Comparación de la actividad de los machos entre distintos meses para ambas parcelas en base a la máxima actividad media registrada en cada mes mediante el test de chi-cuadrado. ${ }^{* * *}: \mathrm{p}<0,001$. NS: no significativo

MAYO JUNIO JULIO AGOSTO SEPTIEMBRE

$\begin{array}{rllllll} & \text { Abril } & \text { NS } & * * * & * * * & * * * & * * * \\ \text { Parcela AR } & \text { Mayo } & & * * * & * * * & * * * & * * * \\ & \text { Junio } & & & \text { NS } & \text { NS } & \text { NS } \\ & \text { Julio } & & & & \text { NS } & \text { NS } \\ & \text { Agosto } & & & & & \text { NS }\end{array}$

\begin{tabular}{clccccc} 
& & MAYO & JUNIO & JULIO & AGOSTO & SEPTIEMBRE \\
\hline \multirow{5}{*}{ Parcela AB } & Abril & $* * *$ & $* * *$ & $* * *$ & $* * *$ & $* * *$ \\
& Mayo & $* * *$ & $* * *$ & $* * *$ & $* * *$ & $* * *$ \\
& Junio & & & NS & NS & NS \\
& Julio & & & & NS & NS \\
& Agosto & & & & & NS
\end{tabular}


Los machos están activos gran número de horas, sobre todo entre las 10 y las $18 \mathrm{~h}$. En cambio en el mes de junio la actividad es muy baja, registrándose porcentajes del 10 al $20 \%$ como máximo. Según avanza el verano se observa un aumento de actividad aunque poco notable y no significativo. A principios de abril la actividad se hace mayor a las horas centrales del día, cuando las temperaturas son más altas. En mayo la actividad tiende a hacerse bimodal y durante el verano parecen estar más activos por la mañana. Ya desde principios de abril los machos patrullan su área y cortejan a las hembras. La mayoría de las cópulas tiene lugar durante la primera quincena de junio, según muestran las huellas de mordiscos en los costados de las hembras.

\section{ACTIVIDAD DE HEMBRAS}

Las figuras 3 y 4 muestran los resultados referentes a las hembras. En estas no se observan diferencias tan acusadas entre meses como en los machos. En la parcela AB las diferencias entre meses no son significativas y en la $\mathbf{A R}$ son escasas (Cuadro 2). Nuestros resultados indican que su actividad diaria ocupa menor número de horas. La cantidad de hembras activas a una hora determinada es baja, registrándose porcentajes máximos entre el 10 y el $29 \%$. Durante los meses de abril y mayo las hembras están activas en las horas centrales del día, en junio la actividad es básicamente matinal y en julio y agosto se hace bimodal.

\section{CUADRO 2}

Comparación de la actividad de hembras entre distintos meses en la parcela $\mathbf{A R}$ en base a la máxima actividad registrada en cada mes mediante el test de chi-cuadrado. ${ }^{* *}: \mathrm{p}<0,01$. NS: no significativo

MAYO JUNIO JULIO AGOSTO SEPTIEMBRE

$\begin{array}{llllll}\text { Abril } & \text { NS } & \text { NS } & \text { NS } & \text { NS } & - \\ \text { Mayo } & & * * & \text { NS } & * * & - \\ \text { Junio } & & & * * & \text { NS } & - \\ \text { Julio } & & & & * * & - \\ \text { Agosto } & & & & & -\end{array}$

\section{ACTIVIDAD DE SUBADULTOS}

Los subadultos presentan nula actividad (parcela AR) o muy escasa (parcela AB) en abril, donde se observan como mucho el 3\%/ de los individuos una hora determinada (Figuras 5 y 6). Las diferncias entre el mes de abril y los demás son significativas en ambas parcelas. En cambio, entre los demás meses no hay diferencias (Cuadro 3).

A partir de mayo se observa actividad generalizada de subadultos hasta septiembre. Téngase en cuenta que ya en agosto los censos contabilizan como 


\section{CUADRO 3}

Comparación de la actividad de subadultos entre distintos meses para ambas parcelas en base a la máxima actividad registrada en cada mes mediante el test de chi-cuadrado.

${ }^{* * *}: \mathrm{p}<0,001 .{ }^{* *}: \mathrm{p}<0,01 .{ }^{*}: \mathrm{p}<0,05$. NS: no significativo

MAYO JUNIO JULIO AGOSTO SEPTIEMBRE

$\begin{array}{rlccccc} & \text { Abril } & * * * & * * * & * * * & * * * & * * * \\ \text { Parcela AR Ano } & & \text { NS } & * & \text { NS } & * \\ & \text { Manio } & & & \text { NS } & \text { NS } & \text { NS } \\ & \text { Julio } & & & & * * & \text { NS } \\ & \text { Agosto } & & & & & * *\end{array}$

\begin{tabular}{llccccc} 
& & MAYo & JUNIO & JULIO & AGOSTO & SEPTIEM \\
\hline & Abril & $* * *$ & $* * *$ & $*$ & $* * *$ & $* * *$ \\
Parcela AB AB & Mayo & NS & NS & NS & NS & NS \\
& Junio & & & NS & NS & NS \\
& Julio & & & & $*$ & NS \\
& Agosto & & & & & NS
\end{tabular}

subadultos a los jóvenes, que ya han alcanzado su tamaño y sobreestiman el número de subadultos activos. Prácticamente los subadultos están activos preferentemente a las horas centrales del día y a partir de julio se observan más por la mañana.

\section{ACTIVIDAD DE JOVENES}

Los jóvenes se caracterizan porque sólo una pequeña fracción de ellos están activos a cualquier hora en cualquier mes (Figuras 7 y 8). No se registran diferencias significativas entre meses en ambas parcelas. En abril y mayo están activos a las horas centrales del día y después su actividad es preferentemente matinal. También se caracterizan los jóvenes porque su rango de horas a lo largo del día no es muy extenso. Como hemos dicho al hablar de los subadultos, no hay actividad de jóvenes en agosto, pues aparecen como subadultos en los censos de estos.

\section{COMPARACION ENTRE PARCELAS}

Al comparar la actividad de los lagartos de ambas parcelas entre sí (Cuadro 4) se ve que las diferencias entre jóvenes son inexistentes. En cuanto a hembras y subadultos, sólo se han registrado diferencias significativas en el mes de mayo. En machos se han registrado diferencias significantes durante los meses de abril, mayo, junio y julio. 
En los meses de abril y mayo el porcentaje de machos activos es superior en la parcela $\mathbf{A B}$ mientras que en los meses de junio y julio sucede lo contrario.

\section{CUADRO 4}

Comparación de la actividad entre ambas parcelas en base a la máxima actividad media en cada mes mediante el test de chi-cuadrado. ${ }^{* * *}: \mathrm{p}<0,001{ }^{* *}: \mathrm{p}<0,01 .^{*}: \mathrm{p}<0,05$. NS: no significativo.

\begin{tabular}{lcccc} 
& MACHOS & HEMBRAS & SUBADULTOS & JóVENE \\
\hline Abril & $* * *$ & NS & NS & NS \\
Mayo & $* * *$ & $* *$ & $*$ & NS \\
Junio & $* *$ & NS & NS & NS \\
Julio & $* *$ & NS & NS & NS \\
Agosto & NS & NS & NS & - \\
Septiembre & NS & - & NS & -
\end{tabular}

\section{DISCUSIÓN}

Lacerta schreiberi coincide con otras especies de zonas templadas en que su actividad anual está restringida a la primavera y al verano, extendiéndose desde abril a octubre (PILORGE, 1982) (SAINT GIRONS, 1976, 1977) (VAN NULAND Y STRIJBOSCH, 1981). En Holanda Ios machos de Lacerta agilis y Lacerta vivípara son los primeros en emerger de la invernada (NULAND y STRIJBOSCH, 1981). Los machos de $L$. schreiberi también emergen unos días antes que las hembras aunque se ven más por estar activos en mayor número y durante más horas. Hemos observado que los jóvenes emergen muy pronto, entre machos y hembras.

La casi total inactividad de los subadultos en abril podría ser ventajosa para éstos pues evitarían la predación precisamente cuando los machos se muestran más en lugares expuestos atrayendo a los depredadores. También la inactividad de estos podría ser ventajosa para la población, evitándose interferencias durante la reproducción.

Lacerta schreiberi muestra patrones de actividad e inactividad que dependen del sexo y de la edad. En machos la actividad es muy alta en la época reproductora. En estos meses necesitan patrullar su área y cortejar a las hembras, exponiéndose fuertemente a los predadores. En esta época prima para ellos la reproducción frente al riesgo de la predación. A partir de junio es muy escaso el porcetaje de machos activos. Durante el verano es ventajosa para ellos la inactividad, evitando la depredación. Solamente aumentan su actividad a finales del verano con el fin de acumular reservas con que hacer frente a la invernada.

En las hembras no se registran variaciones de actividad de la primavera al verano. Las hembras deben acumular energías para la puesta durante la primavera y para el crecimiento y acumulación de grasas durante el verano. En 
ellas la actividad es más baja que en machos pues es más importante para este sexo evitar la depredación y asegurar la puesta. En las hembras el esfuerzo reproductor se reparte equilibradamente de abril a agosto mientras que en los machos el esfuerzo es exclusivamente primaveral.

Los patrones de actividad e inactividad en adultos de $L$. schreiberi coinciden con los dos de Sceloporus virgatus (ROSE, 1981), sugiriendo que estos patrones sean comunes a otras especies de saurios diurnos en áreas equivalentes. En cuanto a los subadultos, sus porcentajes de actividades son altos en verano, indicando que en ellos prima alcanzar cuanto antes la madurez reproductora. La escasa pero continuada actividad encontrada en jóvenes sugiere que en ellos parece más importante evitar la predación. 

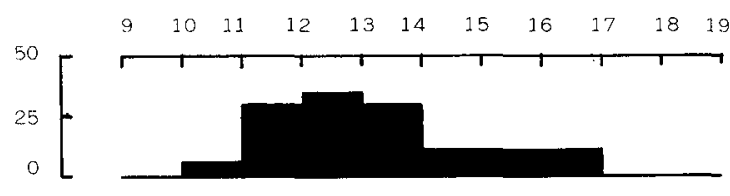

10 ABRIL

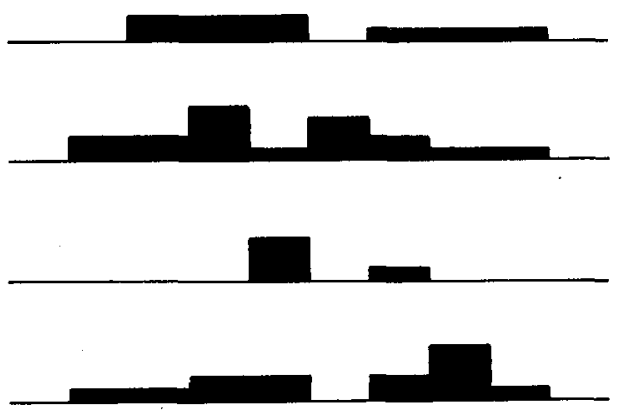

18 ABRIL

25 ABRIL

7 MAYO

10 MAYO
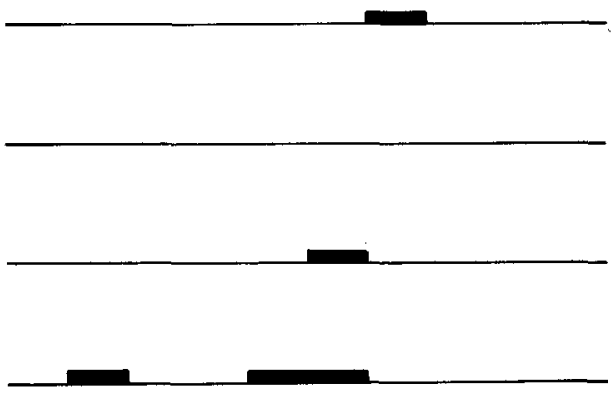

4 JULIO

12 JULIO

17 AGOSTO

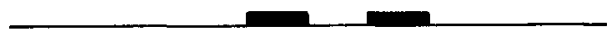

28 Agosto

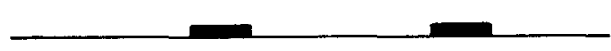

9 SEPTIEMBRE

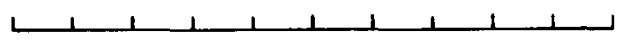

Figura 1.-Proporción de machos activos de la parcela AR a distintas horas según censos realizados de abril a septiembre. 


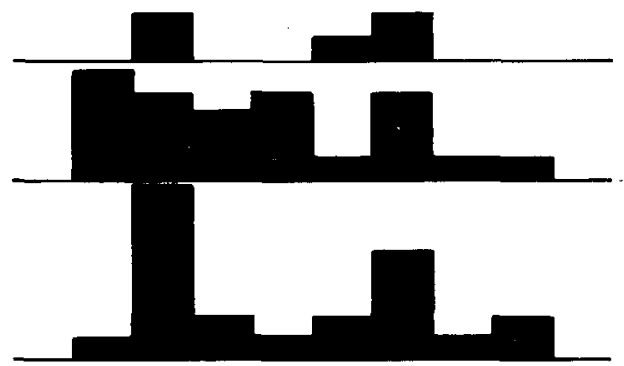

19 ABRIL

27 ABRIL

8 MAYO

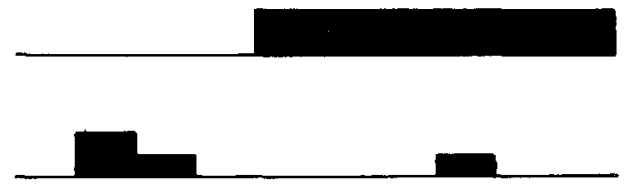

29 MAYO

13 JUNIO
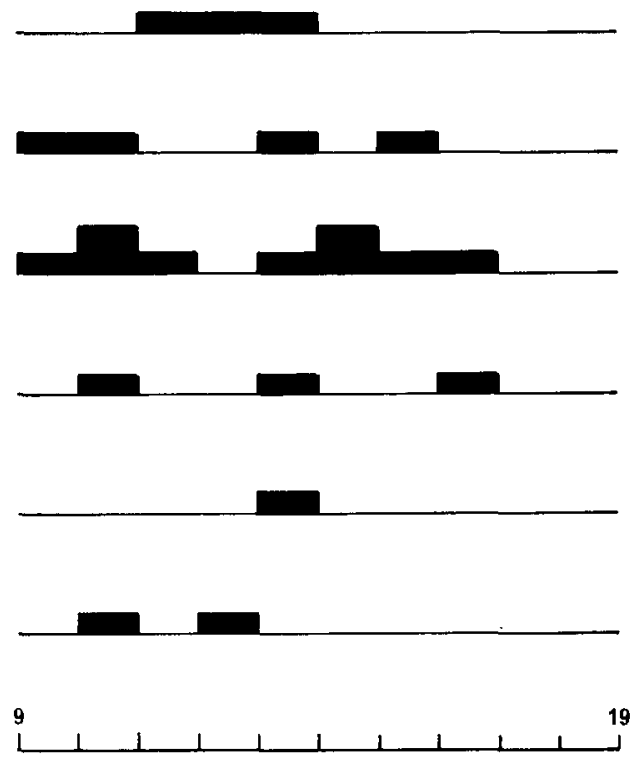

Figura 2.-Proporción de machos activos de la parcela $\mathrm{AB}$ a distintas horas según censos realizados de abril a septiembre. 

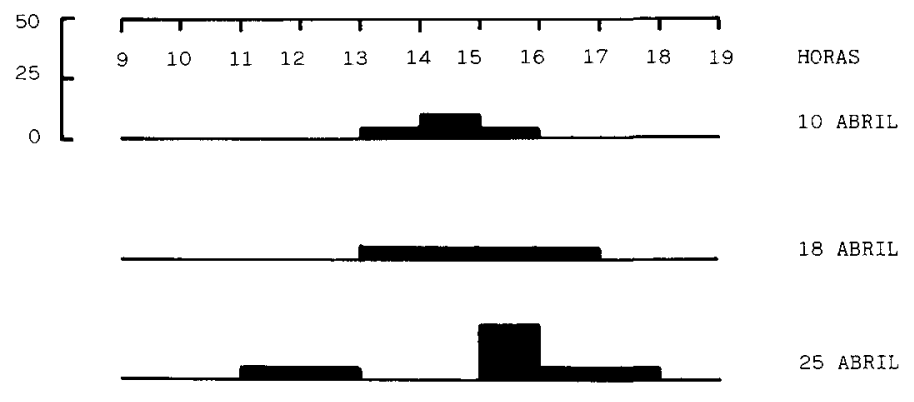

18 ABRIL

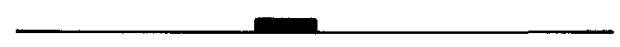

7 MAYO

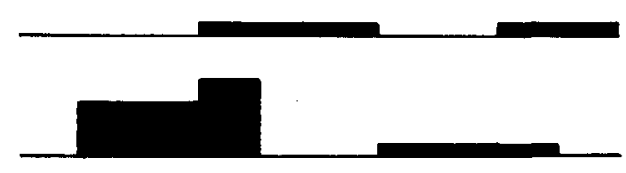

$10 \mathrm{MAYO}$

11 JUNIO

15 JUNIO

28 JUNIO

4 JULIO

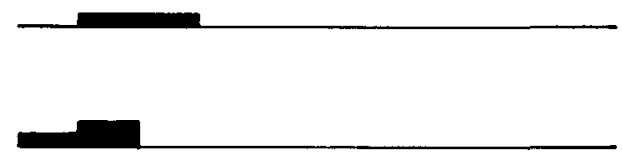

$13 \mathrm{JULIO}$

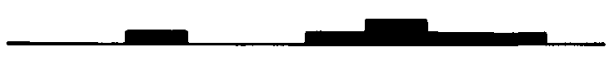

17 AgOSTO

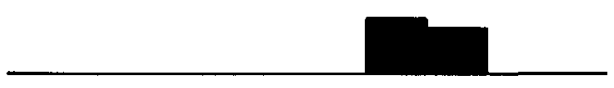

28 AGOSTO

9 SEPTIEMBRE

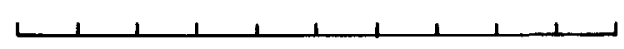

Figura 3.-Proporción de hembras activas de la parcela AR a distintas horas según censos realizados de abril a septiembre. 

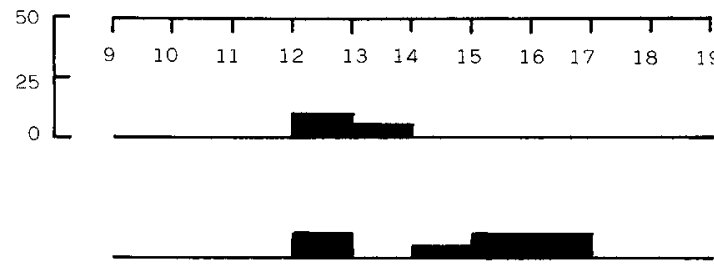

19 ABRIL

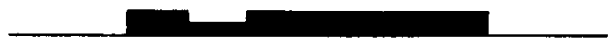

27 ABR.IL

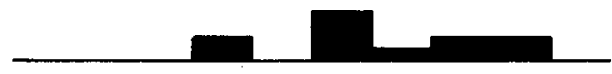

8 MAYO

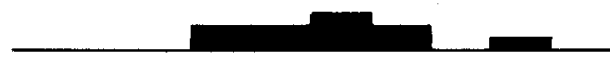

29 MAYO
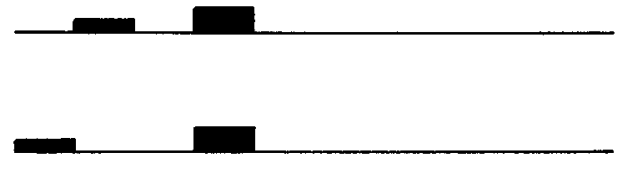

26 JUNIO

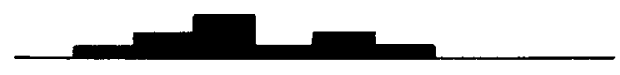

30 JUNIO

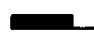

-

8 JULIO

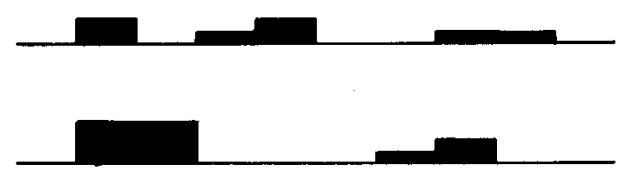

17 JULIO

25 AGOSTO

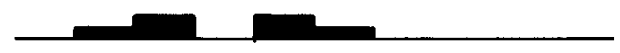

31. AGOSTO

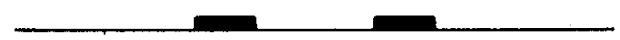

11 SEPTIEMBRE

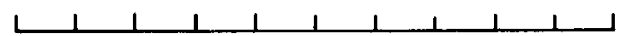

Figura 4.-Proporción de hembras activas de la parcela $\mathrm{AB}$ a distintas horas según censos realizados de abril a septiembre. 

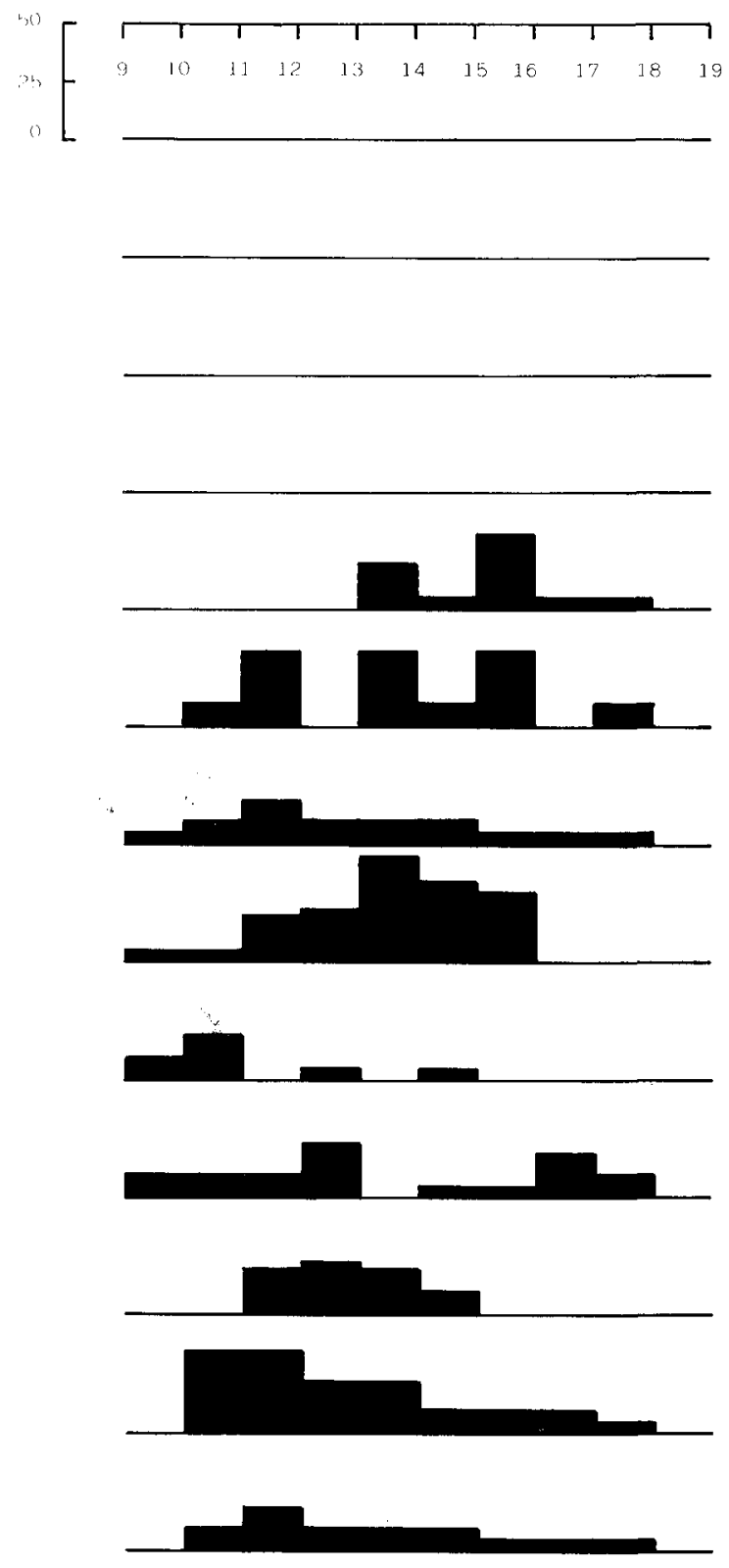

9 SEPTIEMBRE

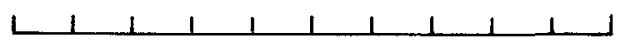

Figura 5.-Proporción de subadultos activos de la parcela.AR a distintas horas según censos realizados de abril a septiembre. 

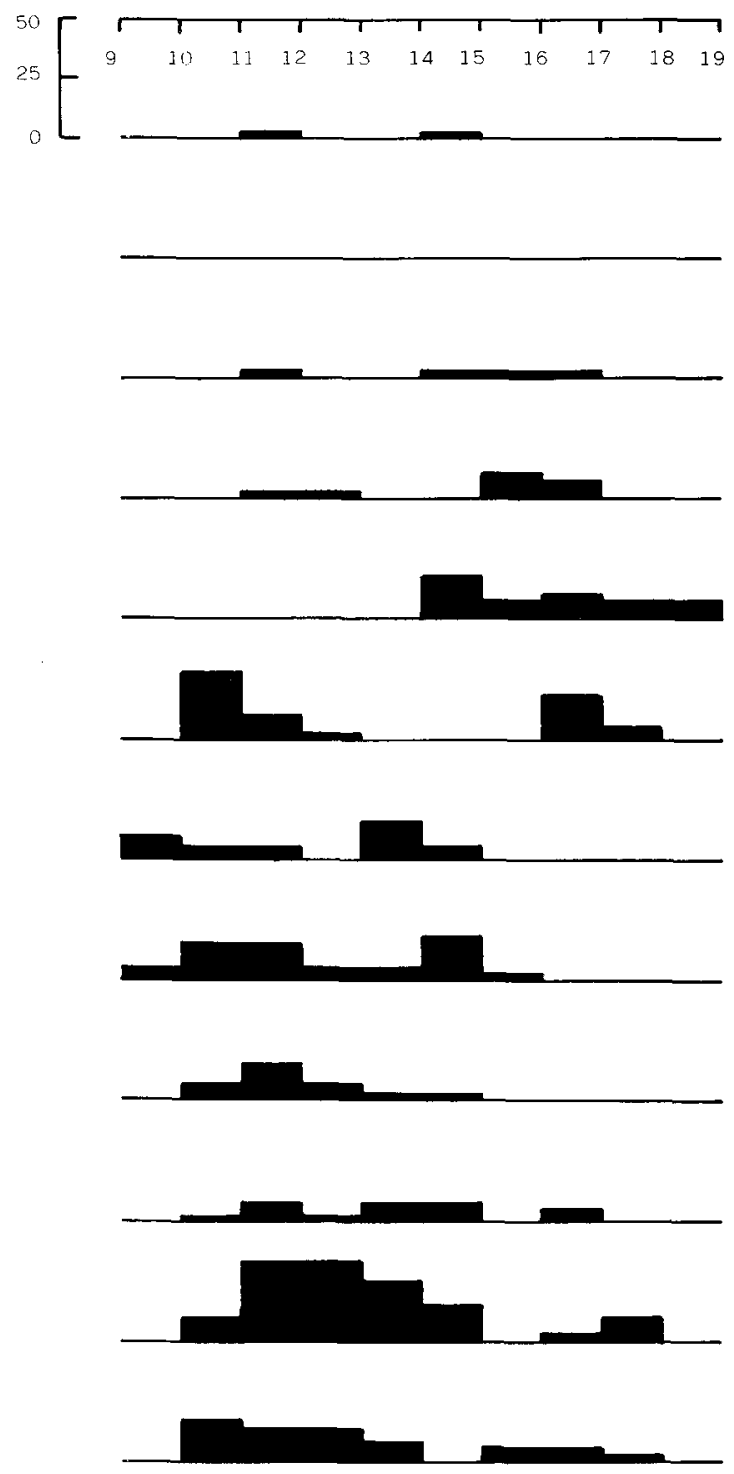

31 Agosto

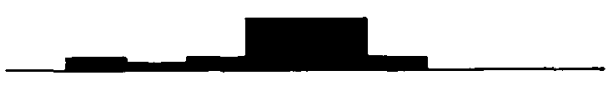

11 SEPTIEMERE

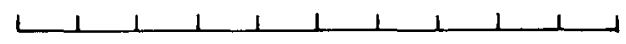

Figura 6.-Proporcion de subadultos activos de la parcela $\mathrm{AB}$ a distintas horas según censos realizados de abril a septiembre. 

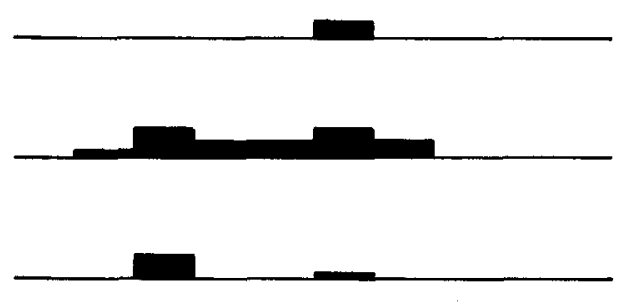

7 MAYO
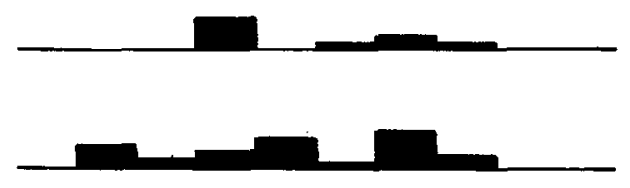

11. JUNIO
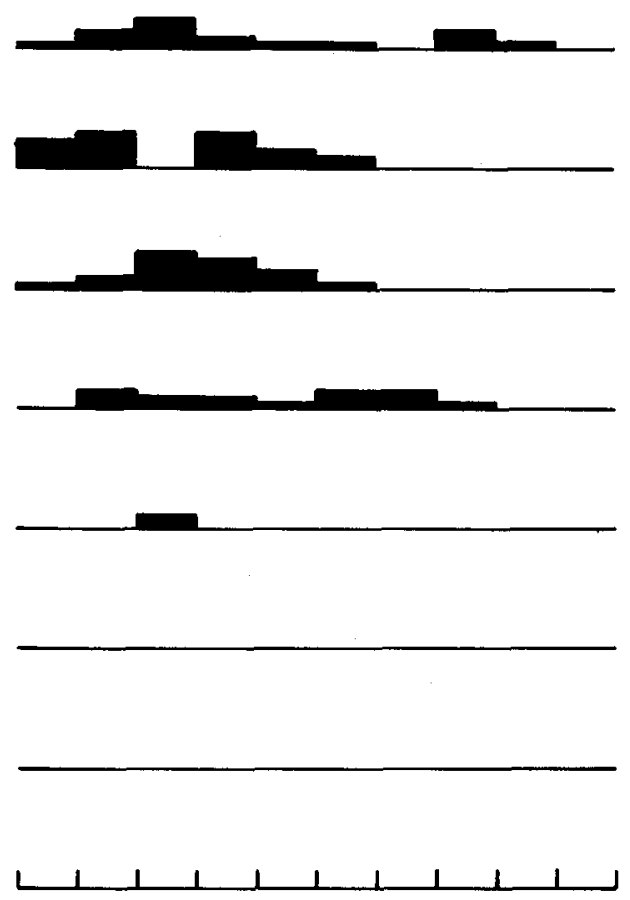

Figura 7.-Proporción de jóvenes activos de la parcela AR a distintas horas según censos realizados de abril a septiembre. 

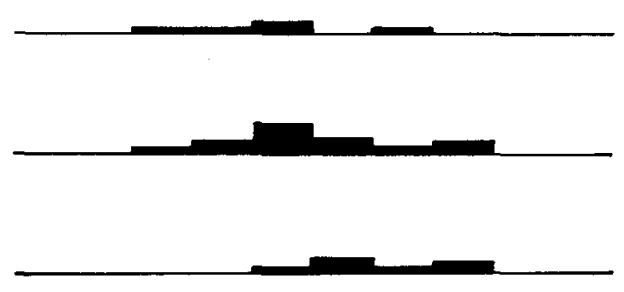

8 MAYO
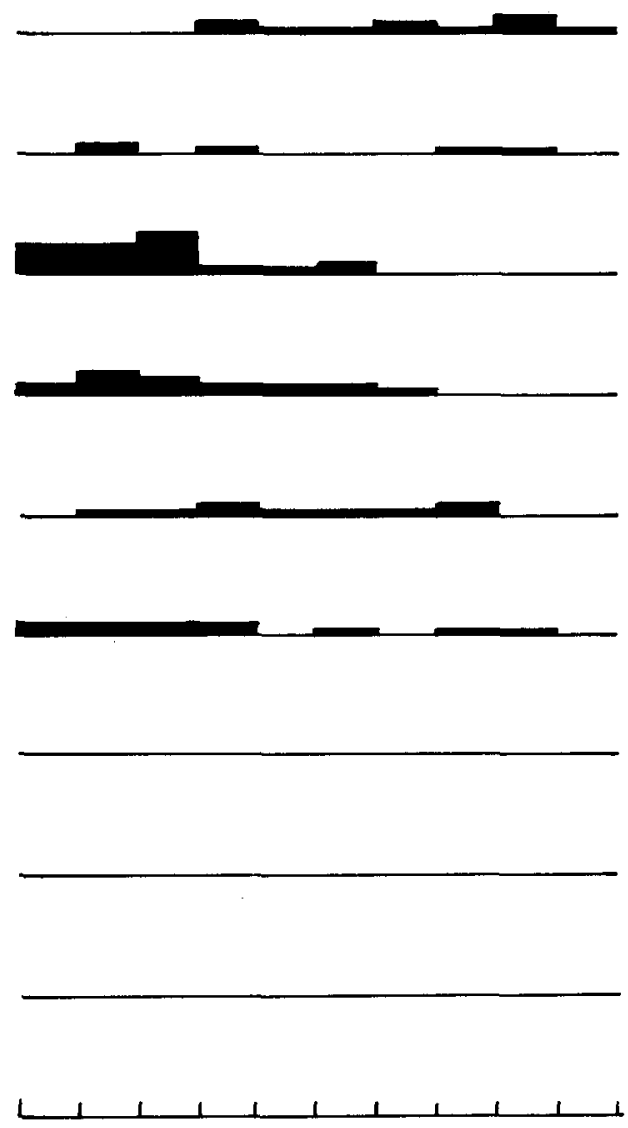

Figura 8.-Proporción de jóvenes activos de la parcela $\mathrm{AB}$ a distintas horas según censos realizados de abril a septiembre. 


\section{BIBLIOGRAFIA}

BUSACK, S. D. 1976: Activity cycles and body temperatures of Acanthodactylus erythrurus. Copeia, 1976: 826-830.

CREUSERE, F. M. y WHITFORD, W. G. 1982: Temporal and spatial resource partitioning in a chihuahuan desert lizard community. pp. 121-127. En: SCOTT, N. J. (ED). Herpetological communities. U. S. Fish Wildl. Serv., Wildl. Res. Rep. No. 13.

PEREZ MELLADO, V. 1983: Activity and thermoregulation patterns in two species of Lacertidae: Podarcis hispanica (Steindachner, 1870) and Podarcis bocagei (Seoane, 1884). Cienc. Biol. Ecol. Syst. 5: 5-12.

PILORGE, T. 1981: Structure et dynamique d'une population du lezard vivipare. E. N. S. Publ. Lab. Zool. No. 18.

ROSE, B. 1981: Factors affecting activity in Sceloporus virgatus. Ecology, 62: 706-716.

SAINT GIRONS, M. C. 1976: Relations interspecifiques et cycle d'activité chez Lacerta viridis et Lacerta agilis (Sauria, Lacertidae). Vie Milieu, 26: 115-132.

SAINT GIRONS, M. C. 1977: Le cicle d'activité chez Lacerta viridis et ses rapports avec la structure sociale. Terre Vie, 31: 101-116.

SEVA, E. 1982: Taxocenosis de lacértidos en un arenal costero alicantino. Universidad de Alicante. $317 \mathrm{p}$.

SIMON, C. A. y MIDDENDORF, .G A. 1976: Resource partitioning by an iguanid lizard: temporal and microhabitat aspects. Ecology, 57: 1317-1320.

VAN NULAND, C. J. y STRIJBOSCH, H. 1981: Annual rhytmics of Lacerta vivipara Jacquin and Lacerta agilis agilis Linné (Sauria, lacertidae) in the Netherlands. Amphibia-Reptilia, 2: $83-95$. 